\title{
Clinical Study of Changmaile II Combined with Estrogen and Progesterone in Preventing and Treating Intrauterine Adhesion
}

\author{
Qian Liu ${ }^{1 *}$, Xueli Chen² \\ ${ }^{1}$ Department of Obstetrics and Gynecology, The Second People's Hospital Affiliated to Fujian University of Traditional Chinese \\ Medicine, Fuzhou, China \\ ${ }^{2}$ Fujian University of Traditional Chinese Medicine, Fuzhou, China \\ Email: ^rachel_0123@163.com
}

How to cite this paper: Liu, Q. and Chen, X.L. (2021) Clinical Study of Changmaile II Combined with Estrogen and Progesterone in Preventing and Treating Intrauterine Adhesion. Chinese Medicine, 12, 29-35. https://doi.org/10.4236/cm.2021.122004

Received: March 24, 2021

Accepted: June 8, 2021

Published: June 11, 2021

Copyright $\odot 2021$ by author(s) and Scientific Research Publishing Inc. This work is licensed under the Creative Commons Attribution International License (CC BY 4.0).

http://creativecommons.org/licenses/by/4.0/ (c) (i) Open Access

\begin{abstract}
Purpose: To explore the clinical efficacy of Changmaile II combined with cyclic estrogen/progesterone therapy in treating hypomenorrhea caused by intrauterine adhesion (IUA) and its effect on the endometrium after the separation procedure for intrauterine adhesion. Methods: Sixty patients with IUA confirmed by hysteroscopy in the Second People's Hospital of Fujian University of Traditional Chinese Medicine from March 2020 to December 2020 were selected and randomly divided into the control group and the observation group, with 30 cases in each group. After the separation procedure for intrauterine adhesion, the observation group underwent the cyclic estrogen/progesterone therapy alone, while the observation group was administered the in-hospital preparation of Changmaile II in addition to the therapy of the observation group. Menstrual recovery, endometrial thickness, and the diagnostic grading scores of IUA after 3 menstrual cycles were measured in both groups. Results: After the treatment for 3 menstrual cycles, the total effective rate was higher in the observation group (73.33\%) than in the control group $(53.33 \%)(\mathrm{P}<0.05)$. The period flow was heavier in the observation group than in the control group $(\mathrm{P}<0.05)$. The endometrial thickness of both groups was thicker than before treatment $(\mathrm{P}<0.05)$, nonetheless the difference between the groups was not statistically significant $(P>0.05)$. After treatment, the diagnostic grading scores of IUA were higher in the observation group than in the control group $(\mathrm{P}<0.05)$. Conclusions: Changmaile II combined with estrogen and progesterone artificial cycle therapy could increase the menstrual volume and endometrial thickness of patients with intrauterine adhesion, while promoting the endometrial repair, with better efficacy than artificial cycle therapy alone.
\end{abstract}




\section{Keywords}

Intrauterine Adhesion, Changmaile II, Estrogen/Progesterone

\section{Introduction}

Intrauterine adhesion (IUA) refers to mutual adhesion between the uterine walls, with partial or complete closure of the uterine cavity, caused by damage to the basal layer of the endometrium due to factors such as uterine cavity operations and infections [1]. It can be manifested as extremely light period flow, amenorrhea, periodic lower abdominal pain, secondary infertility, and adverse pregnancy outcomes such as spontaneous abortion, recurrent abortion, and placental implantation abnormalities. With the increase of various uterine cavity operations and the widespread use of hysteroscopy, the incidence and diagnosis rate of IUA has increased year by year in China [2]. It is reported that the incidence of IUA will increase from $10 \%$ to $30.6 \%$ after one or more curettages [3]. At present, IUA is mainly treated by hysteroscopic lysis, but the incidence of postoperative adhesion recurrence is about $30 \%$ and can reach $62.5 \%$ in severe patients [4], resulting in an unsatisfactory efficacy. Therefore, how to effectively promote endometrial repair and prevent adhesion recurrence is a problem that needs to be solved urgently.

In traditional Chinese medicine (TCM), there is no such disease as "intrauterine adhesion". Instead, according to its different clinical manifestations, it is attributed to "hypomenorrhea", "amenorrhea", "infertility", or "recurrent miscarriage". However, TCM has unique advantages in improving menstrual disorders and infertility. Previous studies on the characteristics of TCM syndrome elements of IUA have shown that blood stasis is the most common nature syndrome element of IUA [5]. In this study, Changmaile II, a TCM capsule for removing blood stasis and dredging collaterals, was used in combination with cyclic estrogen/progesterone therapy to prevent adhesion recurrence, promote endometrial repair, and improve the period flow, menstrual cycles, and period blood color. The report is as follows.

\section{Materials and Methods}

\subsection{Subjects}

A total of 60 patients with IUA, who had been admitted to the Department of Gynecology, the Second People's Hospital of Fujian University of Traditional Chinese Medicine from March 2020 to December 2020, were randomly divided into the control group and the observation group, with 30 in each group. There were no statistically significant differences in the age, course, and the number of uterine cavity operations between the two groups $(P>0.05)$, and they were comparable (Table 1). This study protocol was approved by the hospital ethics committee. 
Table 1. Comparison of general information between the two groups $(\bar{x} \pm s, \mathrm{n}=30)$.

\begin{tabular}{cccc}
\hline Group & Age (years) & Course (months) & Number of uterine cavity operations \\
\hline Control & $27.2 \pm 5.5$ & $6.5 \pm 3.5$ & $3.2 \pm 1.1$ \\
Observation & $26.5 \pm 6.5$ & $7.5 \pm 3.3$ & $3.0 \pm 1.35$ \\
P value & 0.81 & 0.90 & 0.85 \\
\hline
\end{tabular}

\subsection{Diagnostic Criteria}

Hysteroscopy is the gold standard for diagnosing IUA. According to An Atlas of Hysteroscopy [6] and Chinese Obstetrics \& Gynecology [7], the diagnostic criteria was drafted as follows: hysteroscopy shows the membranous adhesions crisscrossed between the endometriums, or a muscular adhesion formed between newly formed fibrous tissues and smooth muscles, or the fibrous connective tissue adhesion formed by the tough scar tissue, with partial or complete closure of the uterine cavity.

\subsection{Inclusion Criteria}

Meets the diagnostic criteria for IUA; aged between 18 and 45 years old; has informed consent for this study.

\subsection{Exclusion Criteria}

Endocrine and metabolic diseases including congenital adrenal hyperplasia, androgen-secreting tumors, Cushing syndrome, and thyroid disease; use of tamoxifen or estrogen and progesterone for more than 3 months; malignant tumors of the reproductive system; complicated serious diseases of the respiratory, digestive, circulatory, nervous, and blood systems.

\subsection{Treatment Methods}

Both groups underwent hysteroscopic separation procedure for intrauterine adhesion. Estrogen and progesterone artificial cycle therapy started on the first day after surgery: Take one estradiol tablets/estradiol dydrogesterone tablet (trade name: Femoston 2/10mg, Abbott Biologicals, Netherlands; registration number: H20150345; Serving size: $2 \mathrm{mg}+2 \mathrm{mg}: 10 \mathrm{mg}$ ) orally every day, for three consecutive courses of treatment, with 28 days as a course. On the basis of the above treatment, patients in the observation group were given Changmaile II (drug approval number: Z05104026), consisting of leeches and earthworms, 5 capsules each time, three times a day, for a total of 12 weeks (stopped during menstruation).

\subsection{Observation Measures}

Both groups underwent secondary hysteroscopy three menstrual cycles after treatment. The diagnostic grading scores of IUA, menstrual volume scores, and endometrial thickness were compared before and after treatment. The IUA 
scores were assessed based on the consensus of Chinese experts in clinical diagnosis and treatment of IUA (2015). The period flow was evaluated using the Pictorial Blood Assessment Chart (PBAC). The endometrial thickness was measured on the 15th day of the menstrual cycle using transvaginal ultrasound.

\subsection{Efficacy Evaluation}

According to the Guiding Principles for Clinical Research of New Chinese Medicines (Trial) [8], the efficacy was evaluated as follows: 1) Cured: Both the menstrual cycles and the period flow are normal; hysteroscopy shows that the external shape of the uterine cavity is normal, the inner membrane surface is smooth, and the bilateral uterine horns or fallopian tube openings are clearly visible; 2 ) Effective: The period flow is less than normal, and hysteroscopy shows that the shape of the uterine cavity is basically normal, with some adhesions still visible; 3) Ineffective: The menstrual indicators have not recovered, and hysteroscopy shows that the shape of the uterine cavity has not changed compared with before treatment.

\subsection{Statistical Methods}

SPSS 20.0 statistical software was used for data analysis. Measurement data were expressed as mean \pm standard deviation, and $t$ test was used for comparison between groups. Count data were expressed as rate (n, \%), and the comparison between groups is performed by $\chi^{2}$ test. $\mathrm{P} \leq 0.05$ was considered statistically significant.

\section{Results}

\subsection{Comparison of Clinical Efficacy between the Two Groups}

The total effective rate was higher in the observation group (73.33\%) than in the control group $(53.33 \%)(\mathrm{P}<0.05)$, as shown in Table 2.

\subsection{Comparison of IUA Scores between the Two Groups before and after Treatment}

There was no statistically significant difference in the diagnostic grading scores of IUA between the two groups before treatment $(P>0.05)$. The IUA scores of both groups after treatment were lower than those before treatment $(\mathrm{P}<0.05)$. After treatment, the score of IUA was higher in the observation group than in the control group $(\mathrm{P}<0.05)$, as shown in Table 3.

Table 2. Comparison of clinical efficacy between the two groups $[\mathrm{N} / \mathrm{n}(\mathrm{p} / \%)]$.

\begin{tabular}{cccccc}
\hline Group & $\mathrm{N}$ & Cured & Effective & Ineffective & Total effective rate (\%) \\
\hline Control & 30 & 6 & 10 & 14 & $53.33^{\mathrm{a}}$ \\
Observation & 30 & 9 & 13 & 8 & $73.33^{\mathrm{a}}$ \\
\hline
\end{tabular}

a. Comparison between the two groups, $\mathrm{P}<0.05$. 
Table 3. Comparison of IUA scores between the two groups before and after treatment $(\bar{x} \pm s$, s/points).

\begin{tabular}{cccc}
\hline Group & $\mathrm{N}$ & Before & After \\
\hline Control & 30 & $11.82 \pm 3.82$ & $7.80 \pm 4.12^{\mathrm{ab}}$ \\
Observation & 30 & $10.70 \pm 3.96$ & $6.59 \pm 3.62^{\mathrm{ab}}$ \\
\hline
\end{tabular}

aa. Comparison with before treatment, $\mathrm{P}<0.05$. b. Comparison between groups, $\mathrm{P}<0.05$.

\subsection{Comparison of Endometrial Thickness between the Two Groups}

The endometrial thickness of both groups was measured by transvaginal ultrasound before treatment and on the 15th day of the menstrual cycle after treatment. There was no statistically significant difference in the endometrial thickness between the two groups before treatment $(\mathrm{P}>0.05)$. Compared with before treatment, the endometrial thickness of both groups increased after treatment $(\mathrm{P}$ $<0.05)$, but the difference between the two groups was not statistically significant $(\mathrm{P}>0.05)$, as shown in Table 4.

\subsection{Comparison of Menstrual Volume Scores between the Two Groups before and after Treatment}

As shown in Table 5, before treatment, the difference in menstrual volume scores between the two groups was not statistically significant $(P>0.05)$. After treatment, menstrual volume scores of both groups were higher than before treatment $(\mathrm{P}<0.05)$, and the difference was statistically significant $(\mathrm{P}<0.05)$.

\section{Discussion}

IUA is a fibrotic disease, which is caused by curettage or other uterine cavity operations usually, the endometrial injury reaches the basal layer and the abnormal inflammatory response promotes the occurrence of fibrosis. Similar to other fibrotic diseases, its clinical treatment is very difficult. The hysteroscopic lysis of IUA can only partially or completely restore the anatomy of the uterine cavity. Current postoperative interventions include the auxiliary uses of hormone drugs, drugs that improve endometrial blood flow, intrauterine barrier, and stem cells [9], and their efficacy is unsatisfactory. For this reason, postoperative prevention of adhesion recurrence, promotion of endometrial regeneration and repair, and ultimately improvement of fertility outcomes has gradually become research hotspots.

TCM believes that the cause of IUA is mostly that the metal blade directly damages the blood network of the uterus. The results of previous studies showed that the most common location syndrome element of IUA is the uterus, and the nature syndrome element of blood stasis is most frequently found in different degrees of IUA. This shows that the location of IUA is largely the uterus, and its basic pathological change is blood stasis. The blood stasis blocks the uterus, the veins and collaterals are thus blocked, and the menstrual flow cannot go down; if 
Table 4. Comparison of endometrial thickness between the two groups before and after treatment $(\bar{x} \pm s, \mathrm{~d} / \mathrm{mm})$.

\begin{tabular}{cccc}
\hline Group & $\mathrm{N}$ & Before & After \\
\hline Control & 30 & $5.40 \pm 1.60$ & $6.54 \pm 1.47^{\mathrm{a}}$ \\
Observation & 30 & $5.34 \pm 1.43$ & $6.94 \pm 1.62^{\mathrm{a}}$ \\
\hline
\end{tabular}

a. Comparison with before treatment, $\mathrm{P}<0.05$.

Table 5. Comparison of menstrual volume scores between the two groups before and after treatment ( $\bar{x} \pm s, \mathrm{~s} /$ points).

\begin{tabular}{cccc}
\hline Group & $\mathrm{N}$ & Before & After \\
\hline Control & 30 & $6.85 \pm 3.80$ & $10.80 \pm 5.12^{\mathrm{ab}}$ \\
Observation & 30 & $7.35 \pm 3.66$ & $13.59 \pm 5.45^{\mathrm{ab}}$ \\
\hline
\end{tabular}

aa. Comparison with before treatment, $\mathrm{P}<0.05$. b. Comparison between groups, $\mathrm{P}<0.05$.

the thoroughfare and controlling vessels are occluded, one will not be able to get pregnant; for a long time, new blood will not be generated, and there will be insufficiency of qi and blood in the thoroughfare and controlling vessels, which will be unable to nourish the uterus. Therefore, the author proposed that the method of promoting blood circulation to remove blood stasis and dredge collaterals should be used throughout IUA treatment. Changmaile II is an in-hospital preparation composed of leeches and earthworms. Effective in promoting blood circulation, removing blood stasis, and dredging collaterals, it is widely used in the clinical treatment of diseases with the syndrome of blood stasis. Particularly, leeches can remove blood stasis and dredge meridian to eliminate symptoms. The Holy Husbandman's Classic on Roots and Herbs describes that it "eliminates blood stasis and facilitates water channels". Moreover, the mobile and penetrating nature of earthworms was used to make it effective in dredging the meridian. The Holy Husbandman's Classic on Roots and Herbs states that the leeches can "eliminate evil blood and stasis", and Zhang Xichun believes that "the leech ranks first among the blood-breaking drugs". The two drugs are insect-type drugs for dredging the collaterals, with mobile and penetrating nature. They are what $\mathrm{Wu}$ Jutong described as "the mobile ones that pass through the blood in the collaterals to penetrate and break any stasis". In addition, Changmaile II is crushed with raw medicinal materials into capsules, which make up for the fishy and foul smell of earthworms and leeches that are difficult to take orally.

The results of this study showed that the total effective rate was higher in the treatment group (73.33\%) than in the control group (53.33\%), and the menstrual recovery was better than the control group. The endometrial thickness of both groups was thicker than before treatment. The diagnostic grading scores of IUA were higher in the treatment group than in the control group after treatment. All of these indicated that Changmaile II combined with estrogen and progesterone artificial cycle therapy could effectively reduce the incidence of re-adhesion, im- 
prove menstrual conditions, and increase the effective rate. This method makes use of the advantages of TCM, increases the preventing and treating measures for IUA, and is expected to improve the clinical efficacy.

\section{Acknowledgements}

We are grateful to the women who agreed to participate in research for this study.

\section{Found}

Traditional Chinese Medicine Scientific Research Project of Fujian Health Department (2017FJZYJC205).

\section{Conflicts of Interest}

The authors declare no conflicts of interest regarding the publication of this paper.

\section{References}

[1] March, C.M. (2011) Asherman's Syndroms. Seminars in Reproductive Medicine, 29, 83-94.

[2] Obstetrics and Gynecology Branch of Chinese Medical Association. (2015) Consensus of Chinese Experts on Clinical Diagnosis and Treatment of Intrauterine Adhesions. Chinese Journal of Obstetrics and Gynecology, 50, 881-887.

[3] Hooker, A.B., de Leeuw, R., van de Ven, P.M., Bakkum, E.A., Thurkow, A.L., Vogel, N.E.A., van Vliet, H.A.A.M., Bongers, M.Y., Emanuel, M.H., Verdonkschot, A.E.M., et al. (2017) Prevalence of Intrauterine Adhesions after the Application of Hyaluronic Acid Gel after Dilatation and Curettage in Women with at Least One Previous Curettage: Short-Term Outcomes of a Multicenter, Prospective Randomized Controlled Trial. Fertility and Sterility, 107, 1223-1231. https://doi.org/10.1016/j.fertnstert.2017.02.113

[4] Chen, Y., Liu, L., Luo, Y., et al. (2017) Effects of Aspirin and Intrauterine Balloon on Endometrial Repair and Reproductive Prognosis in Patients with Severe Intrauterine Adhesion: A Prospective Cohort Study. BioMed Research International, 2017, Article ID: 8526104. https://doi.org/10.1155/2017/8526104

[5] Liu, Q., Chen, X.L., Huang, S.P., et al. (2020) Study on Correlation between Signs of Uterine Cavity under Hysteroscopy and TCM Syndrome Elementsin Intrauterine Adhesion. Journal of Traditional Chinese Medicine, 27, 19-23.

[6] Xia, E.L. (2016) Hysteroscopy and Atlas. 3rd Edition, Henan Science and Technology Press, Zhengzhou, 189-192.

[7] Cao, Z.Y. (2004) Chinese Obstetrics and Gynecology. 2nd Edition, People's Medical Publishing House, Beijing, 2406.

[8] Zheng, X.Y. (2002) Guiding Principles for Clinical Research of New Chinese Medicine (Trial). China Medical Science and Technology Press, Beijing.

[9] Yan, X.Z. (2020) Research Advances in Prevention of Readhesion after Transcervical Resection of Adhesions. Luzhou Medical College, 43, 429-433. 